\title{
Interaction between a Broad-spectrum Antibiotic and Silver Nanoparticles in a Human Gut Ecosystem
}

\author{
Das $P^{1 \# *}$, Saulnier $E^{1 \#}$, Carlucci $C^{2}$, Allen-Vercoe $E^{2}$, Shah $V^{3}$ and Walker VK $K^{1,4 *}$ \\ ${ }^{1}$ Department of Biology, Queen's University, Kingston, ON K7L 3N6, Canada \\ ${ }^{2}$ Department of Molecular and Cellular Biology, University of Guelph, Guelph, ON N1G 2W1, Canada \\ ${ }^{3}$ College of the Sciences and Mathematics, West Chester University, West Chester, PA 19382, USA \\ ${ }^{4}$ Department of Biomedical and Molecular Sciences, and School of Environmental Studies, Queen's University, Kingston, ON K7L 3N6, Canada \\ \#Co-first authors
}

\begin{abstract}
The antimicrobial properties of engineered silver nanoparticles (AgNPs) have led to their wide use in diverse consumer products. Ampicillin too, acts as a broad-spectrum antibiotic and thus is prescribed for the treatment of many common infections, but with the problematic emergence of ampicillin-resistant bacteria. As a consequence, there has been some interest in the combination of these two distinct chemistries prompted by the clinical challenge of resistance. Prior to trials of combination therapy, however, it is important to understand the impact on human microbiomes. Here we investigated the effect of ampicillin and AgNPs, both individually and in a combined therapy on a human intestinal ecosystem known as a defined experimental community (DEC-60). The DEC-60 consortia was co-treated with a concentration of AgNPs (50 mg/L) known to have a minimal impact, and a broad range of the antibiotic up to the clinical dose $(6 \mathrm{mg} / \mathrm{L})$. The addition of AgNPs to sub-clinical doses of ampicillin $(0.06$ and $0.6 \mathrm{mg} / \mathrm{L})$ had a significantly impact $(\mathrm{p}<0.001-0.05)$ on gas production $\left(\% \mathrm{CO}_{2}\right.$ and $\left.\% \mathrm{~N}_{2}\right)$ and changed the composition of fatty acid methyl ester signatures so that they were distinct from each individual antimicrobial, as well as un-treated control cultures. DNA sequencing, supported by multidimensional scaling analysis, confirmed the community shift and showed distinct phylogenetic distributions at different concentrations of ampicillin, depending on the presence of AgNPs. Together, these results suggest that the antibacterial efficacy of sub-clinical doses of ampicillin was increased by AgNPs, possibly due to the synergistic effect of damage to the bacterial cell walls. Not only does this analysis provide insight into AgNP toxicity, it offers some promise that combined antimicrobial therapies may have value in a clinical setting.
\end{abstract}

Keywords: Silver nanoparticles; Ampicillin; Human gut consortium; Sequencing; Fatty acid methyl esters; Anaerobic metabolism

\section{Introduction}

The known antimicrobial effects of engineered silver nanoparticles (AgNPs), combined with increasing efficient production methods have contributed significantly to their prevalence in diverse consumer goods ranging from food items and packaging, to sporting equipment, clothing, water purification units and cosmetics [1-3]. Surface charges associated with some AgNPs preparations may contribute to their toxicity [4]. However, multiple studies have highlighted the critical role of nano-size dependent effects compared to ionic silver, with an emphasis on the unique properties of AgNPs [5-7]. Although there are reports of mammalian cell cytotoxicity [8-10], these effects are likely relatively minimal at the concentrations used for antimicrobial activity [11]. Biofilms, which provide a refuge for Gram positive and negative bacteria, appear to be also inhibited by AgNPs [11]. Taken together, these observations have helped foster the use of AgNPs for medical and personal health applications. However, even though these concentrations of AgNPs may not be directly toxic to human tissues, the efficacy of AgNPs against bacteria can result in shifts in bacterial consortia, which in turn could have an impact on host organisms [e.g. 12-14]. Certainly, community changes in response to these NPs have been noted in intestinal flora subsequent to oral ingestion in a range of species including fish, insects, and in a human model gut ecosystem $[5,7,15]$.

The human microbiome is a diverse system composed of 100200 species per individual [16], with the majority ( 90\%) falling into Bacteroidetes (generally staining Gram negative) and Firmicutes (generally staining Gram positive) phyla. Most of the remaining species belong to the Actinobacteria and Proteobacteria [17,18]. Suiting the intestinal microenvironment, resident human gut microbes are either facultatively or obligately anaerobic [19]. It is difficult to accurately recapitulate fecal ecosystems in a reproducible manner because of variations in the ecosystem abundance profiles as influenced by diet and transient microbial presence. However, one solution to this problem has been to create model fecal communities from diverse strains isolated from single donor fecal samples [20]. One such model community, Defined Experimental Ecosystem Consortium-60 members (DEC60 ), has been developed as a proxy for a healthy fecal ecosystem and displays similar diversity at the phylum level to that found generally in human stool samples, and as well specifically includes species that are commonly found in the healthy human gut [21]. A subset of DEC60 strains has been successfully used as a therapeutic ecosystem in the treatment of clinically-challenging Clostridioides difficile infections as an alternative to antibiotic therapy [21].

The persistent and pervasive use of antibiotics both therapeutically in human medicine, and as growth promoters in livestock agriculture has led to the widespread and unsettling emergence of antibiotic resistant bacteria [22]. It is generally agreed that antibiotic resistance is a significant threat to human health and that restriction of their

*Corresponding author: Virginia $\mathrm{K}$ Walker and Pranab Das, Department of Biology, Queen's University, Biosciences Complex, Canada; Tel: 6135336123; Fax 6135336617; E-mail: walkervk@queensu.ca,pd50@queensu.ca

Received October 26, 2016; Accepted October 28, 2016; Published October 31, 2016

Citation: Das P, Saulnier E, Carlucci C, Allen-Vercoe E, Shah V, et al. (2016) Interaction between a Broad-spectrum Antibiotic and Silver Nanoparticles in a Human Gut Ecosystem. J Nanomed Nanotechnol 7: 408. doi: 10.4172/2157. 7439.1000408

Copyright: (c) 2016 Das P, et al. This is an open-access article distributed under the terms of the Creative Commons Attribution License, which permits unrestricted use, distribution, and reproduction in any medium, provided the original author and source are credited. 
use may help preserve their efficacy [23,24]. AgNP treatment, or a combined use of AgNPs with antibiotics, could reduce dosage thresholds for therapeutic activity and may also be effective against multi-drug resistant bacteria [25-28]. Indeed, this is the reason that AgNPs are currently being tested in a number of experimental pointof-use treatment systems for hospitals and nursing homes [29-31].

Here we have used the DEC-60 model microbiome system to investigate changes induced by both AgNPs and ampicillin treatment, both individually and in a combined therapy. Our goal was to examine if there were any synergistic effects of the combination therapy. There is some urgency to these experiments considering the increasing use of AgNPs in medical settings and the unknown impacts on the human intestinal microbiome. We hypothesized that co-treatment of the model ecosystem with AgNPs and ampicillin could perturb the system in a manner distinct from each individual antimicrobial. Ideally, combinatorial treatment with both amendments would allow a reduction in the dosage of the broad-spectrum antibiotic, ampicillin, in order to decrease the probability of the development of resistance to this drug.

\section{Materials and Methods}

\section{Silver nanoparticle and microparticle characterization}

Suspended AgNPs, capped with poly(vinyl)pyrrolidone (PVP) were purchased from nanoComposix (San Diego, CA; DAG1964). Capping reduces agglomeration as well as the release of silver ions into the medium. The manufacturer reported diameters of $10.6 \pm 1.7$ $\mathrm{nm}(1.0 \mathrm{mg} / \mathrm{mL}$ stock concentration). The AgNPs were independently characterized via determination of number-based size distributions, dynamic light scattering (DLS), and zeta potential with a Malvern Zetasizer (Malvern Instruments Ltd., UK). As well, although nominal stock concentrations for AgNPs were provided by the manufacturer, the actual ${ }^{107} \mathrm{Ag}$ concentration for the stock AgNPs suspension was confirmed by inductively coupled plasma-mass spectrometry (ICP-MS; XSeries IIl; Thermo Scientific, Germany), optimized using PlasmaLab software, with $5 \mu \mathrm{g} / \mathrm{L}$ stock solutions of silver and indium as internal standards, as described [30]. Suspensions (50 mg/L) of AgNPs with each concentration of ampicillin used experimentally $(0.06,0.6,3$, and $6 \mathrm{mg} / \mathrm{L}$ ) were further analyzed for size by transmission electron microscopy (TEM; Hitachi H-7000, Japan). To control for silver ions, powders of silver microparticles (7.5 $\mu \mathrm{m}$; AgMPs) were purchased from MK Nano (MK Impex Canada, Mississauga, Canada). Suspension of these non-functionalized AgMPs were also characterized by TEM as reported elsewhere [7].

\section{Human gut ecosystem}

A defined experimental community prepared with a total of 60 different bacterial isolates, designated as DEC-60, was cultured as described using an Infors Multifors bioreactor system (Infors, Switzerland) converted into a chemostat for continuous culture [21]. Nitrogen was bubbled through the system to maintain anaerobiosis. To mimic conditions of the human distal colonic microenvironment, the temperature was maintained at $37^{\circ} \mathrm{C}$, and at a $\mathrm{pH}$ of 6.9-7.0. The autoclaved single-stage chemostat media, a mixture of insoluble starches, bile salts, mucin and other microbial growth supporting components was prepared, added to the culture vessel, reduced, and inoculated with freshly-cultured DEC-60 components, which had been each cultured separately on Fastidious Anaerobe Agar (Acumedia) plates containing $5 \%$ defibrinated sheep blood (Hemostat laboratories). The vessel was run in batch with $\mathrm{pH}$ control for 1 day prior to running under flow conditions (media fed at a rate of $400 \mathrm{~mL} /$ day) to give a retention time of 24 hours. Steady-state, as assessed through the amplification of $16 \mathrm{~S}$ rRNA gene sequences using polymerase chain reaction (PCR) and denaturing gradient gel electrophoresis (DGGE-PCR) with moving window analysis [32,33], was attained by 7 days following chemostat operation. At steady-state, samples were aseptically removed from the vessel into a Ruskin anaerobic chamber (The Baker Co., USA) with a gas composition of $80 \% \mathrm{~N}_{2}, 10 \% \mathrm{CO}_{2}$, and $10 \% \mathrm{H}_{2}$ and used within $\sim 3$ $\mathrm{h}$ for further experiments.

\section{Treatment groups}

Prior to experimentation, all materials were placed in the anaerobic chamber for several hours to ensure that oxygen was excluded from the cultures [21]. While continually maintaining anaerobic conditions, the subsamples of DEC-60 inocula were seeded into sterile growth medium (1:10). Diluted aliquots $(5 \mathrm{~mL})$ were then placed in sterile glass serum bottles ( $50 \mathrm{~mL}$; Sigma-Aldrich) with treatment-groups established in triplicate, with antibiotic concentration and the presence or absence of nanoparticles as the primary variables. Ampicillin (Sigma Aldrich) was added separately to the serum bottles to a final concentration of $0.06,0.6,3$, and $6 \mathrm{mg} / \mathrm{L}$, both with and without AgNPs. Ampicillin concentrations were based on the use of $6 \mathrm{mg} / \mathrm{L}$ as a concentration approximating the clinical dose used for infections [34], and others at subclinical doses, with the lowest concentration representing two orders of magnitude below the clinical dose. The separate addition of AgNPs to a final concentration of $50 \mathrm{mg} / \mathrm{L}$ was chosen based on our previous observations of a minimal AgNP-mediated impact at this concentration [5]. Control treatment-groups consisted of unamended 1:10 DEC-60, Ag NPs (50 mg/L) in 1:10 DEC-60, and a control for the silver treatment consisting of $50 \mathrm{mg} / \mathrm{L}$ AgMPs in 1:10 DEC-60. Treated serum bottles were sealed with sterilized $20 \mathrm{~mm}$ rubber stoppers and subsequently fitted with WheatonTM aluminum seals (Fisher Scientific). Bottles were then packed in airtight bags with GaspakTM EZ anaerobic gas pouches (Fisher Scientific), and placed in a styrofoam container maintained at $\sim 37^{\circ} \mathrm{C}$. They were subsequently incubated in the dark at $37^{\circ} \mathrm{C}$ for $48 \mathrm{~h}$, while shaken at $\sim 15 \mathrm{rpm}$ (Bigbill Thermolyne shaker; Dubuque, USA).

\section{Gas chromatography}

After incubation, head-space gas samples were collected from each serum bottle. Luer-Lok ${ }^{\mathrm{TM}}$ Tip BD syringes $(10 \mathrm{~mL})$ equipped with $22 \mathrm{G} 1$ Precision Glide needles (Becton Dickinson \& Co., USA) were used to collect gas samples $(5 \mathrm{~mL})$. The samples were immediately injected into an Agilent Technologies 7890B Gas Chromatograph (GC; USA) using a split-less mode, equipped with a stainless steel column $(50 \mathrm{~m} \times 0.53$ $\mathrm{mm}$ internal diameter, $10 \mu \mathrm{m}$ film thickness). Gas chromatography was conducted with the following operational controls: helium $(\mathrm{He})$ gas carrier was set at a rate of $15 \mathrm{~mL} / \mathrm{min}$, the thermal conductivity detector (TCD) was at $250^{\circ} \mathrm{C}$, and oven temperature was maintained at $32^{\circ} \mathrm{C}$ for $1 \mathrm{~min}$. To correspond with the gas signatures of the consortium bacteria [35], peak areas for both $\mathrm{N}_{2}$ and $\mathrm{CO}_{2}$ were analyzed to determine headspace composition using ChemStation Integration Software (Agilent Technologies; USA).

\section{Fatty acid methyl ester profile analysis}

After incubation, aliquots (three $1 \mathrm{~mL}$ samples) of liquid culture were removed from each of the three replicates for all treatment groups. These aliquots were centrifuged at 2,000 $\mathrm{xg}$ for $10 \mathrm{~min}$. The supernatant was aspirated and pellets were preserved at $-80^{\circ} \mathrm{C}$ until fatty acid methyl ester analysis was conducted (Keystone Labs, Canada). MIDI Sherlock Microbial Identification System (Microbial ID Inc., USA) was used to 
extract the fatty acids from bacterial phospholipids in the pellets [36]. Briefly, the pellets were saponified using $1 \mathrm{~mL} \mathrm{NaOH}$ in methanol $(15 \%$ $\mathrm{w} / \mathrm{v}$ ) at $100^{\circ} \mathrm{C}$ for $30 \mathrm{~min}$. Samples were then methylated through the addition of $2 \mathrm{~mL} \mathrm{HCl}$ in methanol $(54 \% \mathrm{w} / \mathrm{v})$ at $80^{\circ} \mathrm{C}$ for $10 \mathrm{~min}$. Fatty acid methyl esters (FAME) were then extracted by phase separation with the addition of $1.25 \mathrm{~mL}$ hexane/methyl-tert-butyl ether $(50 \% \mathrm{v} / \mathrm{v})$ for $10 \mathrm{~min}$. After discarding the aqueous phase, the organic phase was washed with $3 \mathrm{~mL}$ of $0.3 \mathrm{M} \mathrm{NaOH}$ with a few drops of saturated $\mathrm{NaCl}$ for $5 \mathrm{~min}$. Fatty acid species composition was assessed using an Ultra 2 column with flame ionization detector equipped GC (Agilent).

Chromatographic peaks of each sample were then converted to reflect the percentage of each fatty acid species against the total methylated fatty acids.

\section{DNA isolation and sequencing}

After incubation, aliquots $(200 \mu \mathrm{L})$ of the liquid cultures were sampled from each treatment group and DNA was extracted using a QIAamp DNA stool mini kit (Qiagen Sciences, USA), according to the manufacturer's protocol. DNA purity and concentration was assessed initially with an Ultrospec ${ }^{\mathrm{TM}} 1100$ pro UV/visible Spectrophotometer (Amershan Biosciences, USA), and confirmed with a NanoDrop-1000 (Thermo Scientific, USA). Bacterial DNA was further assessed by agarose gel electrophoresis and DGGE-PCR exactly as described previously [5]. These two analyses allowed a second estimate of quantity and qualitatively indicated the presence of multiple bacterial species, respectively. If individual DNA samples were inadequate in that they were not sufficiently pure, would not amplify, or showed evidence of problems after visualization in agarose or did not show multiple bands after DGGE-PCR, the DNA purification was repeated.

Bacterial DNA samples, which were pure and sufficient for subsequent analysis, were subjected to PCR using forward primer, Gray 28F (5'-TTTGATCNTGGCTCAG-3'), and reverse primer, Gray 519r (5'-GTNTTACNGCGGCKGCTG-3'), resulting in the amplification of the V1-V3 hypervariable regions of the 16S rRNA ( 500 bp). PCR was performed with a $50 \mu \mathrm{L}$ reaction mixture containing $4 \mu \mathrm{L}$ of DNA template including $2.5 \mu \mathrm{L}$ of each primer $(10 \mu \mathrm{M}), 5 \mu \mathrm{L}$ of $10 \mathrm{X}$ ViBuffer A (Vivantis), $0.5 \mu \mathrm{L}$ of dNTP mix (10 mM; Thermo Scientific), 2.5 $\mu \mathrm{L}$ of bovine serum albumin $(2 \mathrm{mg} / \mathrm{mL}), 0.4 \mu \mathrm{L}$ of recombinant Taq DNA polymerase $(10 \mathrm{mM}$; Vivantis) and $32.6 \mu \mathrm{L}$ sterile water. PCR conditions were as follows: $5 \mathrm{~min}$ at $94^{\circ} \mathrm{C}$ denaturation, followed by a total of 30 cycles $94^{\circ} \mathrm{C}$ for $1 \mathrm{~min}, 64^{\circ} \mathrm{C}$ for $1 \mathrm{~min}, 72^{\circ} \mathrm{C}$ for $3 \mathrm{~min}$, followed with a final extension at $72^{\circ} \mathrm{C}$ for $7 \mathrm{~min}$. Amplified products (using triplicate samples for each treatment group) were subjected to 454 pyrotag sequencing according to standard protocols (MR DNA, USA). Briefly, products were purified with Agencourt Ampure beads (Agencourt Bioscience, USA) prior to sequencing. Sequencing was conducted using a Genome Sequences FLX System (Roche, USA) with titanium reagents as described [37]. The raw data was then filtered to exclude primers, as well as failed and low quality reads. Chimeras were eliminated using Black Box Chimera Check software (B2C2) $[38,39]$. After de-noising, sequences were clustered using a distributed MegaBLAST.NET algorithm [40], and subsequently compared to published reads in the National Center of Biotechnology Information (NCBI) [41]. Bacterial species designation was determined according to recovered reads in comparison with published database sequences and known identity of members of the DEC-60 consortium.

\section{Statistics}

Statistical analysis of culture gas composition, FAME profiles, and pyrosequencing were conducted by two-way analysis of variance (2F-ANOVA) as well as independent 2 -factor t-tests where appropriate, using the statistics packages R [42] and SPSS (SPSS Inc., USA). Significant differences between pairs of means were evaluated by Tukey's honest significant differences - post-hoc tests. Multidimensional scaling (MDS) of the relative percent composition of $16 \mathrm{~S}$ rRNA sequence was used to reduce the bacterial composition data set and compare multivariate responses to treatments with different concentrations of ampicillin and AgNPs using SAS Ver 9.4 (SAS Institute, USA).

\section{Results}

\section{Electron microscopy of nanoparticles}

Purchased PVP-capped AgNPs were used since as indicated, the capping agent helps prevent agglomeration and previous experiments had shown that no toxicity was attributable to PVP [4]. The AgNPs were analyzed for zeta potential and diameter using DLS and the concentration assessed using plasma-mass spectrometry. The manufacturer's characterization was confirmed with the diameter of the stock AgNPs suspension at $10.9 \pm 1.2 \mathrm{~nm}$ by number-based hydrodynamic size distribution (vs. the supplier's value of $10.6 \pm 1.7$ $\mathrm{nm})$. As well, TEM confirmed that the capped particles remained well dispersed in the experimental media containing $0.06,0.6,3$, and 6 $\mathrm{mg} / \mathrm{L}$ ampicillin (Figure 1A-1D); the particles did not agglomerate and remained within the anticipated size range.

\section{Head-space gas analysis}

The head-space gas volume after $48 \mathrm{~h}$ could not be determined, but the proportion of each gas was achieved using GC analysis. Control (no AgNPs) treatment replicates showed no significant differences in the $\%$ $\mathrm{CO}_{2}$ fraction as compared to AgNPs (Figure 2) and AgMP-treatment groups (data not shown). Similar results were also observed for $\% \mathrm{~N}_{2}$ fractions in controls as compared to AgNPs and AgMPs (Figure 3). Thus the presence of $\mathrm{Ag}$ particles alone appeared to be insufficient to significantly impact bacterial anaerobic respiration (Figure 2). For ampicillin experimental treatments, at a clinical dose $(6 \mathrm{mg} / \mathrm{L})$,
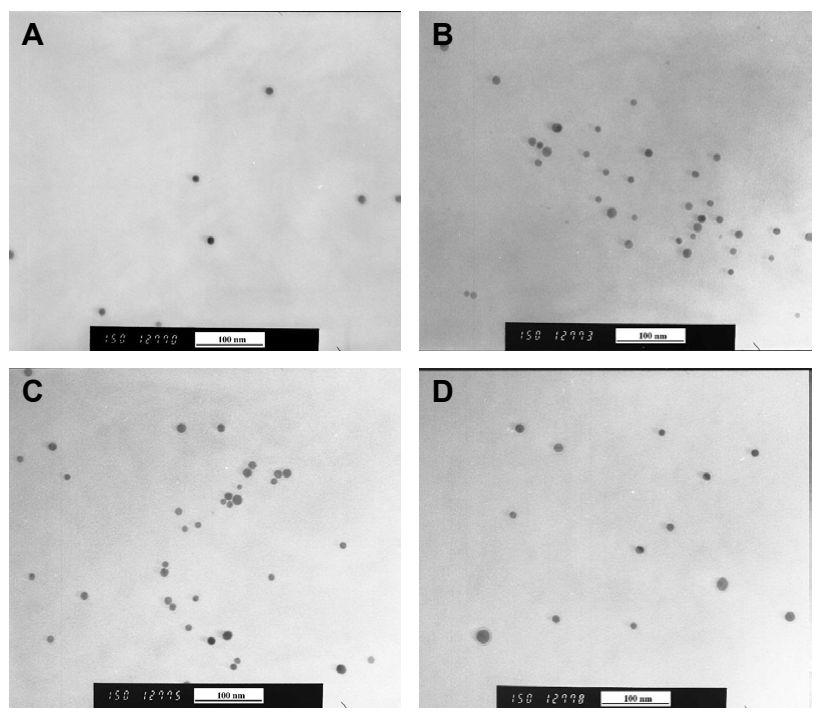

Figure 1: Transmission electron micrographs (TEM) of the silver nanoparticle (AgNPs; $10.9 \pm 1.2 \mathrm{~nm}$ ) preparations used as described in Methods, in the presence of various concentrations of ampicillin (A 0.06, B 0.6, C 3, and D 6 $\mathrm{mg} / \mathrm{L}$ ampicillin). 


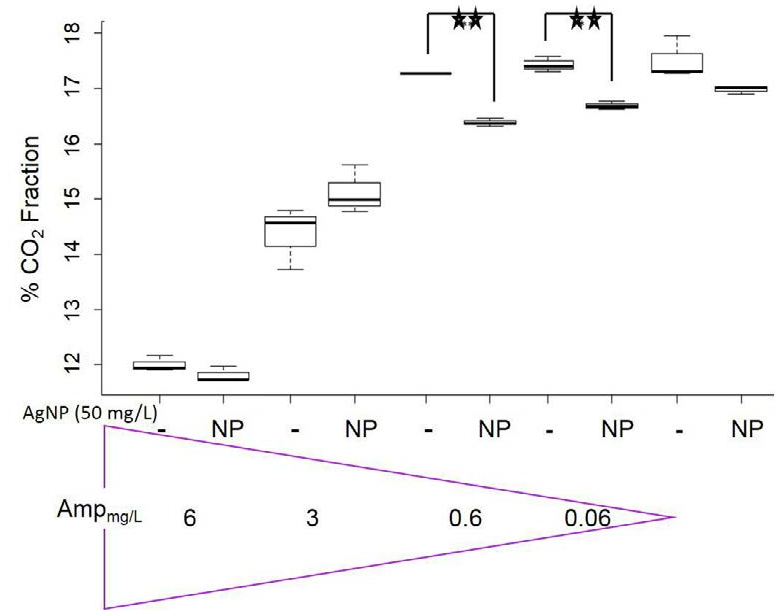

Figure 2: Carbon dioxide $\left(\mathrm{CO}_{2}\right)$ fraction of total culture head-space gas sampled from DEC-60 cultures exposed to silver nanoparticles (AgNPs; $50 \mathrm{mg} / \mathrm{L}$ ) and or ampicillin at the concentrations shown. Gas composition is the mean of each triplicate treatment groups. 'NP' and '-' on the X-axis reflects the presence or absence, respectively, of $50 \mathrm{mg} / \mathrm{LAgNP}$ for a given concentration of ampicillin. The symbol '**' indicates significant pair-wise differences $(p<0.01)$ in the $\mathrm{CO}_{2}$ fraction.

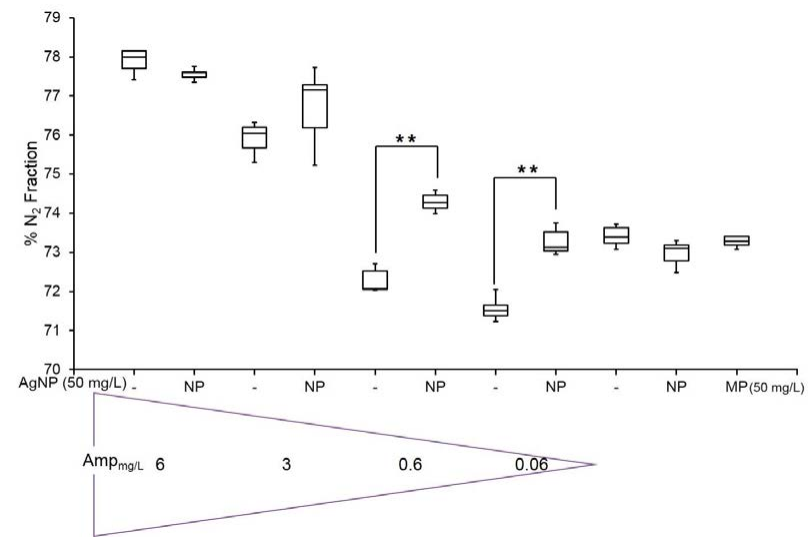

Figure 3: Nitrogen $\left(\mathrm{N}_{2}\right)$ fraction of total culture head-space gas sampled from DEC-60 cultures exposed to silver microparticles (AgMP; $50 \mathrm{mg} / \mathrm{L}$ ), silver nanoparticles (AgNPs; $50 \mathrm{mg} / \mathrm{L}$ ) and/or ampicillin at the concentrations shown. Gas composition is the mean of each triplicate treatment groups. 'NP' and '-' on the X-axis reflects the presence or absence, respectively, of $50 \mathrm{mg} / \mathrm{LAgNP}$ for a given concentration of ampicillin and 'MP' represents AgMP. The symbol '**' indicates significant pair-wise differences $(p<0.01)$ in the $\mathrm{N}_{2}$ fraction.

the percent $\mathrm{CO}_{2}$ was just above the starting gas composition of $10 \%$, suggesting that DEC-60 viability and/or metabolism was severely limited, as expected for the broad-spectrum antibiotic. At sub-clinical ampicillin doses, respiration was elevated relative to that seen at the highest concentration. The percent $\mathrm{CO}_{2}$ and $\mathrm{N}_{2}$ composition were significantly different $(\mathrm{p}<0.01)$ in both 0.06 and $0.6 \mathrm{mg} / \mathrm{L}$ ampicillin treatment groups when compared between treatments, with and without AgNPs (Figures 2 and 3). Summary statistics from 2F-ANOVA revealed significant effects of both AgNPs $(\mathrm{p}<0.01)$ and ampicillin $(\mathrm{p}<0.001)$ on culture head-space percent $\mathrm{CO}_{2}$ and $\mathrm{N}_{2}$ composition, as well as a significant interaction between the two $(\mathrm{p}<0.001)$. Finally, replicates within individual treatments were highly consistent with respect to percent $\mathrm{CO}_{2}$ composition; standard deviations in noncontrol treatments were all $<0.15$, with the exception of treatments at a sub-clinical threshold concentration of $3 \mathrm{mg} / \mathrm{L}$ ampicillin, which showed the highest variation (values of $\sim 0.4 \%$ and $\sim 0.6 \% \mathrm{CO}_{2}$ in the presence and absence of AgNPs, respectively).

\section{Fatty acid profiles}

Examination of FAME profiles revealed a total of 28 unique signatures. Some were present at low levels in all samples and thus these were pooled into a single 'Others' category and therefore only 18 dominant signatures $(\geq 0.15 \mathrm{~mol} \%)$ were considered for subsequent analyses (Figure 4). These were then classified into saturated, Gram positive, Gram negative, and unnamed groups, according to standard conventions [43], but we note that caution should be used in recognizing these groupings since stress can change these classifications [44]. Indeed, we were interested in this aspect of the fatty acid profiles in the various treatment groups. Both relative and total fatty acid abundance were assessed between treatments (Figure 4). Differences in total FAME signatures were significant in low concentration $(0.06 \mathrm{mg} / \mathrm{L})$ ampicillin amendment groups, supplemented with and without AgNPs. They were also significantly different when the $0.6 \mathrm{mg} / \mathrm{L}$ ampicillin and the clinical dose $(6 \mathrm{mg} / \mathrm{L})$ pairs were compared. The $p$-values for the differences between treatments at both control (no ampicillin) and the clinical threshold concentration ( $3 \mathrm{mg} / \mathrm{L}$ ampicillin) were both below the significance limit.

Comparisons of mol\% composition of FAME signatures in AgNP and AgMP control treatments with untreated controls (0 AgNPs and 0 ampicillin) revealed no significant differences for any of the dominant fatty acids assessed (Figure 4). Conversely, comparison of samples treated with the combination treatment of AgNPs and ampicillin vs. AgNP-alone controls (AgCtr) revealed significant differences in percent composition for several fatty acids (Figure 4). For example, in the low antibiotic dose treatment groups, the 17:0 $3 \mathrm{OH}$ signal was absent compared to a mean abundance of $\sim 0.7 \%$ across AgCtr triplicates $(\mathrm{p}<0.05)$. Additionally, in the high dose antibiotic treatment groups, the abundance of several fatty acids was significantly perturbed

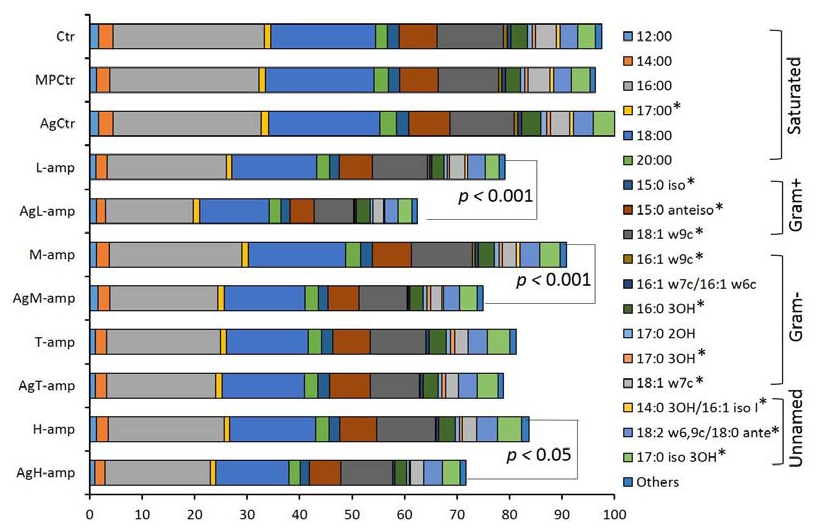

Figure 4: Methylated fatty acids as identified after gas chromatography in DEC-60 cultures (Ctr; no AgNP) or treated with Ag-microparticles (MPCtr), $50 \mathrm{mg} / \mathrm{L}$ silver nanoparticles (AgCtr), and treated with AgNPs supplemented with ampicillin at the following concentrations: 0.06 (L-amp), 0.6 (M-amp), 3 (T-amp), and $6(\mathrm{H}$-amp) $\mathrm{mg} / \mathrm{L}$. They are categorized into saturated, typically Gram-positive (Gram+), and negative (Gram-), as well as unknown signatures (unnamed), but as noted in the results, such groupings should be viewed with caution. FAME results are shown compared to the bench-mark 'AgCtr'treatment (assigned a total abundance of 100). Significant differences in total fatty acid response between treatment groups at the same concentration of ampicillin are indicated, while fatty acid species exhibiting significant differences compared to AgCtr control, are denoted with '“' $(p>0.05)$. 
when compared to AgCtr treatment groups: 18:1 $\omega 9 \mathrm{c}$, a Grampositive associated fatty acid, was increased from $11.8 \%$ to $13.8 \%$ in the presence of $6 \mathrm{mg} / \mathrm{L}$ ampicillin $(\mathrm{p}<0.05), 14: 03 \mathrm{OH} / 16: 1$ iso $\mathrm{I}$, an 'unnamed' species was eliminated in the presence of high doses of antibiotic from $0.8 \%(\mathrm{p}<0.05)$, and another fatty acid, $18: 2 \omega 6,9 \mathrm{c} / 18: 0$ ante, was increased from $3.6 \%$ to $4.8 \%(\mathrm{p}<0.01)$.

\section{AgNPs and ampicillin treatment effects on community composition}

After assigning the 16S rRNA V1-V3 hypervariable region sequences to the bacterial species in DEC-60, those with low sequence abundance in all treatment groups $(\leq 1 \%)$ were pooled into a single 'Others' category. Treatments were then compared for both total sequence reads, a proxy for total bacterial abundance, as well as the relative abundance of each species normalized to the total fatty acids recovered from each experimental treatment, as previously discussed [5] (Figure 5). Significant differences in total response were observed between treatments $( \pm$ AgNPs) at all concentrations of ampicillin (except for control treatments) with $\mathrm{p}<0.01, \mathrm{p}<0.01, \mathrm{p}<0.05$, and $\mathrm{p}<0.05$ for $0.06,0.6,3$, and $6 \mathrm{mg} / \mathrm{L}$ ampicillin treatments, respectively. Large-scale changes in relative species abundance were seen across treatments, with the percentage composition of Bacteroides fragilis decreasing with higher ampicillin doses, and with a corresponding increase in Bacteroides ovatus. Furthermore, MDS analysis of the sequencing results highlighted distinct phylogenetic distributions at each given ampicillin treatment depending on the presence of AgNPs (Figure 6). The tight clustering of replicates by MDS and clear shifts with the changing treatment conditions reflected the dose-dependent bacterial community modulation mediated by the combination AgNP and ampicillin treatment. No significant differences were observed between the AgMPs treatment groups compared to the control without AgNPs (Figure 5).

\section{Discussion}

As a broad-spectrum low cost antibiotic, ampicillin is used orally to treat common infections and because of its utility, it has been placed

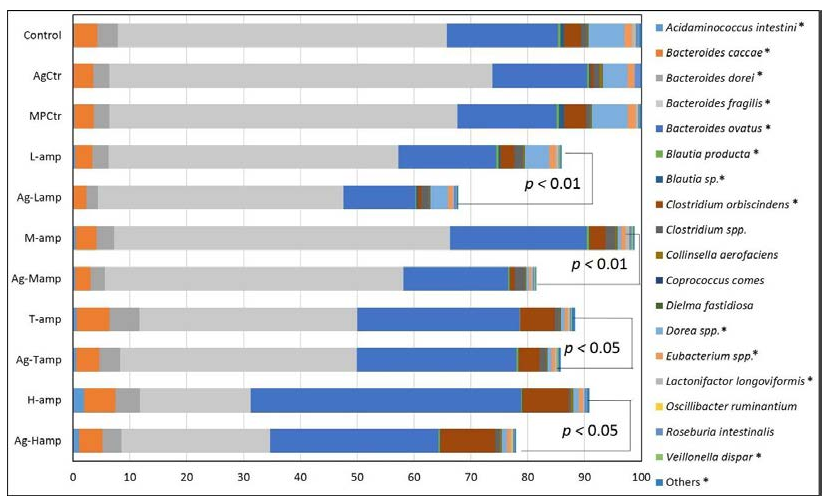

Figure 5: Bacterial community composition of DEC-60 cultures after $48 \mathrm{~h}$ treated with control (no AgNP), $50 \mathrm{mg} / \mathrm{L}$ silver nanoparticles (AgCtr), Ag-microparticles (MPCtr), and ampicillin at the following concentrations: $0.06 \mathrm{mg} / \mathrm{mL}$ (L-amp) and with AgNPs (Ag-Lamp), $0.6 \mathrm{mg} / \mathrm{mL}$ (M-amp) and with AgNPs (Ag-Mamp), $3.0 \mathrm{mg} / \mathrm{mL}$ (T-amp) and with AgNPs (Ag-Tamp), or $6.0 \mathrm{mg} / \mathrm{mL}$ (H-amp) and with AgNPs (Ag-Hamp), all normalized to the total fatty acids recovered in each treatment group. Phylogenetic composition of triplicate cultures for each treatment were pooled, and species with a relative abundance $<1 \%$ were grouped into 'Others'. Significant differences in total abundance between treatments \pm AgNPs are indicated and $p$-values reported. Significant differences between treatments are also denoted with '*' with the legend. on the list of essential medicines by the World Health Organization (http://www.who.int/medicines/events/fs/en/). It is a $\beta$-lactam, which interferes with the assembly of the bacterial cell wall. Not only do these properties make this a valuable antimicrobial but it is also vulnerable to inactivation in resistant bacteria by the production of $\beta$-lactamases, which hydrolyze the $\beta$-lactam ring [45]. Thus, resistance can sometimes be alleviated by the co-administration of drugs that inhibit the hydrolyzing enzymes. Although the antimicrobial mechanism of AgNPs is not known, a popular explanation suggests that interactions between AgNPs and bacteria under aerobic conditions can lead to the production of reactive oxygen species (ROS), which in turn can damage DNA, RNA and proteins [46]. Although we conducted our experiments anaerobically, it is interesting in this regard that our sequencing results showed that facultative anaerobes including Veillonella dispar and Collinsella aerofaciens without ROS-scavenging pathways, either increased in relative abundance or were not different in comparison with treatment groups that lacked AgNPs, respectively (Figure 5). There is also evidence that AgNPs can disrupt bacterial membranes [47]. This mechanism would suggest the potential for a synergistic interaction with the combination of ampicillin and AgNPs, as was observed in our experiments. It is important to note that such interactions may depend critically on the size of the nanoparticle. For example, Habash et al. [48] reported a combination effect of $10 \mathrm{~nm}$ and $20 \mathrm{~nm}$ sized AgNPs when added to the antibiotic aztreonam and tested against Pseudomonas aeruginosa, whereas no interaction was observed with 40, 60 and $100 \mathrm{~nm}$ sized AgNPs. We only tested $10 \mathrm{~nm}$ AgNPs since dose-response data was available for these NPs, and thus it is unknown if larger particles would be less effective; certainly we saw no significant effect with AgMPs.

Over a $48 \mathrm{~h}$ incubation period, the strong antimicrobial effect of ampicillin used at the $6 \mathrm{mg} / \mathrm{L}$ clinical dose was evidenced by negligible gas production likely due to lower metabolic processes or cell death. This is in contrast to the results obtained with the lowest antibiotic doses $(0.6$ and $0.06 \mathrm{mg} / \mathrm{L})$ that showed no difference from unamended controls (Figures 2 and 3). Strikingly, when AgNPs were co-incubated with ampicillin at these same low concentrations there was a significant reduction in produced $\mathrm{CO}_{2}$ (Figure 2). Together, this suggests that cotreatment with ampicillin and AgNP results in a synergistic reduction in targeted bacterial metabolic activity or viability. Furthermore, there was a strong agreement between the total fatty acid responses of each treatment and $\mathrm{CO}_{2}$ production; the increase in respiration was positively correlated $(r=0.64)$ with a shift in the total fatty acid methyl ester profile. Thus, the reduction in total FAME mol\% from the culture substrate, as well as decreased $\mathrm{CO}_{2}$ fermentation suggests an overall reduction in bacterial mass and viability when samples were treated with AgNPs and the higher ampicillin concentrations. Similarly, Birla et al. [49] observed a substantial reduction in metabolic activity when the antibiotic gentamicin was combined with AgNPs and tested against Escherichia coli and Staphylococcus aureus.

While detection of FAME shifts in treated DEC-60 communities revealed unique microbiome profiles for the treatment groups, as indicated, the identification of fatty acids 'signatures' should be treated with caution. Cellular stress, potentially in the form of ampicillin or AgNPs-mediated toxicity, could result in changes to bacterial lipids [50], reducing the reliability of signature benchmarks, but would not affect FAME recovery. Sequencing results, however, also revealed dramatic shifts in the abundance of several key species, suggesting that FAME identifications could be a useful and efficient screen for combinatorial effects. 


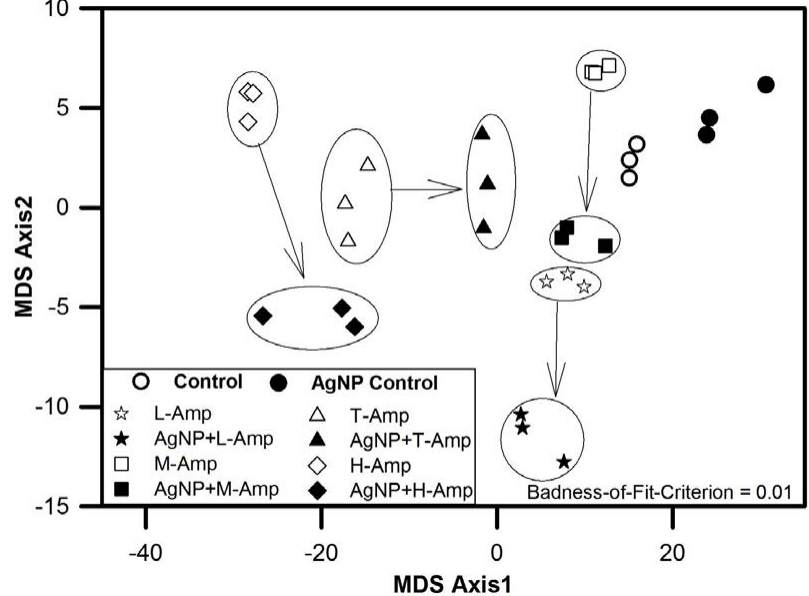

Figure 6: Multidimensional scaling (MDS) plot of DEC-60 consortium community composition based on $16 \mathrm{~S}$ rRNA sequence reads after $48 \mathrm{~h}$ treatment with ampicillin at $0 \mathrm{mg} / \mathrm{mL}$ (Control), $0.06 \mathrm{mg} / \mathrm{mL}$ (L-amp), $0.6 \mathrm{mg} / \mathrm{mL}$ (M-amp), 3.0 $\mathrm{mg} / \mathrm{mL}$ (T-amp), or $6.0 \mathrm{mg} / \mathrm{mL}$ (H-amp) both with and without $50 \mathrm{mg} / \mathrm{LAgNP}$ supplementation. Arrows between clusters indicate shifts between treatment groups with AgNPs from the corresponding group without silver.

Sequencing revealed that the beneficial Bacteroides fragilis was sharply reduced in abundance with increasing ampicillin concentration, however when co-treated with AgNPs, the abundance of this bacterial species remained constant, independent of ampicillin treatment. $B$. fragilis plays a critical role in the human intestinal microbiome, with important roles in immunomodulation, and as a keystone species for overall gut microbial competition.

Consequently, the sustained abundance of this species when treated with AgNPs suggests that co-treatment with NPs and ampicillin while therapeutic, might reduce the inadvertent incidence of gut dysbiosis. In further support of this interpretation, $B$. ovatus, one of the primary constituents of the DEC-60 culture $(\sim 20 \%$ abundance in control conditions) increased in relative abundance to $\sim 53 \%$ when treated with $6 \mathrm{mg} / \mathrm{L}$ ampicillin (thus was not affected by the antibiotic), but this change was abrogated by co-treatment with AgNPs ( $\sim 18 \%$ abundance). Other key commensal species such as Roseburia intestinalis and Coprococcus comes (immune regulation through butyrate secretion [51]), and C. aerofaciens (contributes to host health) maintained or increased in relative abundance when amended with AgNPs.

Notably, the concentration of AgNPs used here was deliberately chosen to have no impact on the gut ecosystem by itself. Nevertheless, our results indicate that the addition of AgNPs to ampicillin treatments have the potential to increase antibiotic efficacy and reduce collateral effects on beneficial intestinal communities. We further suggest that the analysis of synergistic effects may also provide insight into the mechanism of AgNP antimicrobial action. Our results indicate that the observed synergistic antibacterial efficacy of AgNPs combined with ampicillin at different concentrations was nano-size dependent, as also evidenced from the non-significant effect by AgMPs. In addition, we suggest that AgNPs increased the efficacy of sub-clinical doses of 0.06 and $0.6 \mathrm{mg} / \mathrm{L}$ ampicillin at the cell wall by fostering disruption, inhibition of cell-wall synthesis and/or direct damage of it by cross linking of the cell wall peptidoglycan. These experiments thus show promise for combinatory therapeutic treatment with higher concentrations of AgNPs and sub-clinical doses of ampicillin resulting in an overall reduction in the use of this antibiotic.

\section{Acknowledgements}

We thank Dr. G. Cairns (Analytical Services Lab, Queen's University) and Ms. K. Moniz (Queen's University) for assistance with the gas chromatography analyse and DGGE analysis, respectively. Dr. E. Petrof is thanked for her encouragement. This research was supported by an NSERC Discovery grant to VKW and NSERC Discovery Grant to EA-V.

\section{References}

1. Nel A, Xia T, Madler L, Li N (2006) Toxic potential of materials at the nano level. Science 311: 622-627.

2. Sotiriou GA, Pratsinis SE (2010) Antibacterial activity of nanosilver ions and particles. Environmental Science and Technology 44: 5649-5654.

3. Brandt D, Park B, Hoang M, Jacobe HT (2005) Argyria secondary to ingestion of homemade silver solution. Journal of the American Academy of Dermatology 53: S105-S107.

4. Abbaszadegan A, Ghahramani Y, Gholami A, Hemmateenejad B, Dorostkar $S$, et al. (2015) The effect of charge at the surface of silver nanoparticles on antimicrobial activity against Gram-positive and Gram-negative bacteria: A Preliminary Study. Journal of Nanomaterials.

5. Das P, McDonald JAK, Petrof EO, Allen-Vercoe E, Walker VK (2014) Nanosilvermediated change in human intestinal microbiota. Journal of Nanomedicine and Nanotechnology 5: 5.

6. Oberemm A, Hansen U, Bohmert L, Meckert C, Braeuning A, et al. (2016) Proteomic responses of human intestinal Caco-2 cells exposed to silver nanoparticles and ionic silver. Journal of Applied Toxicology 36: 404-413.

7. Han X, Geller B, Moniz K, Das P, Chippindale AK, et al. (2014) Monitoring the development impact of copper and silver nanoparticle exposure in Drosophila and their microbiomes. Sci Total Environ. 487: 822-829.

8. Bohmert L, Niemann B, Lichtenstein D, Juling S, Lampen A (2015) Molecular mechanism of silver nanoparticles in human intestinal cells. Nanotoxicology 9 : 852- 860.

9. Lichtenstein D, Ebmeyer J, Knappe P, Juling S, Böhmert L, et al (2015) Impact of food components during in vitro digestion of silver nanoparticles on cellular uptake and cytotoxicity in intestinal cells. Biological Chemistry 396: 1255-1264

10. Christie M, Andrew Z, Deborah AK, Elizabeth H, Prabir KD, et al. (2015) Oxidative stress-mediated inhibition of intestinal epithelial cell proliferation by silver nanoparticles. Toxicology in Vitro 29: 1793-1808.

11. Sanyasi S, Majhi RK, Kumar S, Mishra M, Ghosh A, et al. (2016) Polysaccharidecapped silver nanoparticles inhibit biofilm formation and eliminate multi-drugresistant bacteria by disrupting bacterial cytoskeleton with reduced cytotoxicity towards mammalian cells. Scientific Reports 6: 16

12. Luo Z, Chen Z, Qiu Z, Li Y, Laing GD, et al. (2014) Gold and silver nanoparticle effects on ammonia-oxidizing bacteria cultures under ammoxidation. Chemosphere 120: 737-742.

13. Das P, Williams CJ, Fulthorpe RR, Hoque ME, Metcalfe CD, et al. (2012) Changes in bacterial community structure after exposure to silver nanoparticles in natural waters. Environmental Sciences and Technology 46: 9120- 9128.

14. Kumar N, Palmer GR, Shah V and Walker VK (2014) The effect of silver nanoparticles on seasonal change in arctic tundra bacterial and fungal assemblages. PLoS ONE 9: e99953.

15. Sarkar B, Jaisai M, Mahanty A, Panda P, Sadique M, et al. (2015) Optimization of the sublethal dose of silver nanoparticle through evaluating its effect on intestinal physiology of Nile tilapia (Oreochromis niloticus L.). Journal of Environmental Science and Health Part a- Toxic/Hazardous Substances and Environmental Engineering 50: 814-823.

16. Avershina E, Rudi K (2015) Confusion about the species richness of human gut microbiota. Beneficial Microbes 6: 5 .

17. Backhed F, Ley RE, Sonnenburg JL, Peterson DA, Gordon JI (2005) Hostbacterial mutualism in the human intestine. Science 307: 1915-1920.

18. Paul BE, Elisabeth MB, Charles NB, Elizabeth P, Les D, et al. (2005) Diversity of the human intestinal microbial flora. Science 308: 1635-1638.

19. O'Hara AM, Shanahan F (2006) The gut flora as a forgotten organ. Embo Reports 7: 688-693.

20. Yen S, McDonald JA, Schroeter K, Oliphant K, Sokolenko S, et al. (2015) 
Citation: Das P, Saulnier E, Carlucci C, Allen-Vercoe E, Shah V, et al. (2016) Interaction between a Broad-spectrum Antibiotic and Silver Nanoparticles in a Human Gut Ecosystem. J Nanomed Nanotechnol 7: 408. doi: 10.4172/2157-7439.1000408

Metabolomic analysis of human fecal microbiota: a comparison of feces-derived communities and defined mixed communities. J Proteome Res 14: 1472-82.

21. Petrof EO, Gloor GB, Vanner SJ, Weese SJ, Carter D, et al. (2013) Stool substitute transplant therapy for the eradication of Clostridium difficile infection: 'RePOOPulating' the gut. Microbiome 1: 3 .

22. Ferrer M, Mendez-Garcia C, Rojo D, Barbas C, Moya A (2016) Antibiotic use and microbiome function. Biochemical Pharmacology.

23. Emerson DC, Luiz FPF, José PO, Marcelo MS, Roberto AO, et al. (2015) Comparison of methods to detect the in vitro activity of silver nanoparticles (AgNP) against multidrug resistant bacteria. Journal of Nanobiotechnology 13: 16 .

24. Walker B, Barrett S, Polasky S, Galaz V, Folke C, et al. (2009) Environment. Looming global-scale failures and missing institutions. Science 325: 1345 -

25. http://www.who.int/mediacentre/factsheets/antibiotic-resistance/en/

26. Gurunathan S (2015) Biologically synthesized silver nanoparticles enhances antibiotic activity against Gram-negative bacteria. Journal of Industrial and Engineering Chemistry 29: 217-226.

27. Panácek A, Smékalováa M, Rová RV, Bogdanová K, Röderová $M$, et al (2016) Silver nanoparticles strongly enhance and restore bactericidal activity of inactive antibiotics against multiresistant Enterobacteriaceae. Colloids and Surfaces B-Biointerfaces 142: 392-399.

28. Tenover FC, Tickler IA, Persing DH (2012) Antimicrobial-resistant strains of Clostridium difficile from North America. Antimicrobial Agents and Chemotherapy 56: 2929-2932.

29. Rajilic-Stojanovic M, Smidt H, de Vos WM (2007) Diversity of the human gastrointestinal tract microbiota revisited. Environmental Microbiology 9 : 2125-2136.

30. Lin S, Huang R, Cheng Y, Liu J, Lau BLT, et al. (2013) Silver nanoparticlealginate composite beads for point-of-use drinking-water disinfection. Water Research 47: 3959-3965.

31. Loo SL, Fane AG, Lim TT, Krantz WB, Liang YN, et al. (2013) Superabsorbent cryogels decorated with silver nanoparticles as a novel water technology for point-of-use disinfection. Environmental Science and Technology 47: 93639371.

32. McDonald JAK, Schroeter K, Fuentes S, Heikamp-dejong I, Khursigara CM et al. (2013) Evaluation of microbial community reproducibility, stability and composition in a human distal gut chemostat model. J Microbiol Methods 95 167-174.

33. Das P, Xenopoulos MA, Williams CJ, Hoque ME, Metcalfe CD (2012) Effects of silver nanoparticles on bacterial activity in natural waters. Environmental Toxicology and Chemistry 31: 122-130.

34. Magni L, Sjövall J, Syvälahti E (1978) Comparative clinical pharmacology of bacampicillin and high oral doses of ampicillin. Infection 6: 283-289.

35. Sahakian AB, Jee SR, Pimentel M (2010) Methane and the gastrointestinal tract. Digestive Diseases and Sciences 55: 2135-2143.
36. Sasser M (2001) Identification of bacteria by gas chromatography of cellular fatty acids, MIDI inc., Sandy Drive Newark, DE 19713.

37. Dowd SE, Callaway TR, Wolcott RD, Sun Y, McKeehan T, et al. (2008) Evaluation of the bacterial diversity in the feces of cattle using 16S rDNA bacterial tag-encoded FLX amplicon pyrosequencing (bTEFAP). BMC Microbiol 8: 125

38. Gontcharova V, Youn E, Wolcott RD, Hollister EB, Gentry TJ, et al. (2010) Black Box Chimera Check (B2C2): a windows-based software for batch depletion of chimeras from bacterial $16 \mathrm{~S}$ rRNA gene datasets. Open Microbiol J 4: 47-52.

39. Bailey MT, Dowd SE, Parry NM, Galley JD, Schauer DB, et al. (2010) Stressor exposure disrupts commensal microbial populations in the intestines and leads to increased colonization by Citrobacter rodentium. Infect Immunol 78: 1509-19.

40. Dowd SE, Zaragoza J, Rodriguez JR, Oliver MJ, Payton PR (2005) Windows. NET network distributed basic local alignment search toolkit (W.ND-BLAST) Bmc Bioinformatics 6: 14.

41. Cole JR, Wang Q, Cardenas E, Fish J, Chai B, et al. (2009) The Ribosomal Database Project: improved alignments and new tools for rRNA analysis. Nucleic Acids Research 37: D141-D145.

42. Team RC (2012) R Foundation for Statistical Computing, Vienna, Austria.

43. Bartelt-Ryser J, Joshi J, Schmid B, Brandi H, Balser T (2005) Soil feedbacks of plant diversity on soil microbial communities and subsequent plant growth. Perspectives in Plant Ecology, Evolution and Systematics 7: 27-49.

44. Suutari M, Laakso S (1994) Microbial fatty acids and thermal adaptation. Crit Rev Microbiol 20: 285-328.

45. Livermore DM (1995) $\beta$-Lactamases in laboratory and clinical resistance. Clin Microbiol Rev 8: 557-584

46. Cabiscol E, Tamarit J, Ros J (2000) Oxidative stress in bacteria and protein damage by reactive oxygen species. Int Microbiol 3: 3-8.

47. Su HL, Chou CC, Hung DJ, Lin SH, Pao IC, et al. (2009) The disruption of bacterial membrane integrity through ROS generation induced by nanohybrids of silver and clay. Biomaterials 9: 5979-5987.

48. Habash MB, Park AJ, Vis EC, Haris RJ, Khursigara CM (2014) Synergy of silver nanoparticles and aztreonam against Pseudomonas aeruginosa PAO1 biofilms. Antimicrob Agents Ch 58: 5818-5830.

49. Birla SS, Tiwari V, Gade AK, Ingle AP, Yadav AP, et al. (2009) Fabrication of silver nanoparticles by Phoma glomerata and its combined effect against Escherichia coli, Pseudomonas aeruginosa and Staphylococcus aureus. Letters in Applied Microbiology 48: 173-179.

50. Gurunathan, S (2014) Rapid biological synthesis of silver nanoparticles and their enhanced antibacterial effects against Escherichia fergusonii and Streptococcus mutans. Arabian Journal of Chemistry.

51. Rivière A, Selak M, Lantin D, Leroy F, De Vuyst L (2016) Bifidobacteria and butyrate-producing colon bacteria: importance and strategies for their stimulation in the human gut. Front Microbiol 7: 979. 